\title{
Uterine rupture in Singapore: Trends and lessons learnt
}

\author{
Lay Kok Tan, ${ }^{1}$ MBBS, FRCOG, MMED (O\&G), FAMS, Suan Tiong $\underline{\text { Beh }},{ }^{2}$ MBBS, MRCOG, FAMS
}

Uterine rupture is arguably one of the most dreaded acute obstetric complications in obstetrics. Synonymous with significant maternal and perinatal morbidity and mortality risks, uterine rupture is usually encountered in the context of vaginal births after previous caesarean section (VBAC).

The paper by Tan et al., ${ }^{1}$ which is a retrospective review of uterine ruptures in a busy obstetric hospital, provides both timely and much useful local data for practising obstetricians. There were 48 uterine ruptures in mostly young multiparous women in the current series, 5 of whom required a hysterectomy. While there were no maternal deaths, there was 1 who was clearly brought to the brink when she developed hypovolaemic shock and cardiac arrest. Perinatal outcomes fared less well with 12 stillbirths and neonatal deaths. That there were no maternal deaths is also a testament not only to the excellent management of these emergencies, but also to the prompt access to multidisciplinary expertise available in a tertiary centre. This may not be so easily accessible in other hospitals, and should therefore be an important consideration for practitioners dealing with VBAC cases.

The study period of 2003-2014 is significant, as this was after the publication of the article by Lydon-Rochelle et al. in The New England Journal of Medicine in 2001,2 which showed that VBAC was associated with an increased rupture risk even if labour presented spontaneously, and was further increased with induced labours, particularly with prostaglandins.

The rupture rate for this epoch was 1 in 3,062, which the authors point out is an increase compared to other Singapore studies done in the seventies ${ }^{3}$ and the eighties. ${ }^{4}$ The results are intriguing, as the article by LydonRochelle et al. had an impact that decreased the rate of VBAC and increased the use of elective repeated caesarean section in developed countries..$^{5}$ This latest Singapore review reports that $25 \%$ of the ruptures occurred in unscarred uteri and hence uterine ruptures are not entirely preventable. More importantly, this paper also shows clearly that there are now other important procedures other than caesarean section that scar the uterus, which in turn can predispose it to rupture.

Previous laparoscopic myomectomy features prominently as an important cause of uterine rupture in the current series. The ruptures are largely characterised by dramatic antepartum presentations via the uterine fundus, with $40 \%$ of these occurring as early as during the second trimester, with unsurprisingly worse maternal and perinatal outcomes compared to intrapartum ruptures. All the laparoscopic myomectomies in the series were performed in other centres with scant detail on closure technique. Clearly a history of previous laparoscopic myomectomy, particularly if surgical details are unavailable, must now be regarded as an obstetric highrisk factor for antepartum rupture, and symptoms of abdominal pain must be regarded with suspicion and thoroughly investigated. These data should also call into question the role of laparoscopic myomectomies for women who have yet to complete their families. Perhaps more importantly, it also highlights the need for adequate surgical training in repairing the resulting uterine defect following myomectomy laparoscopically, to be on par with what is achieved at laparotomy. And above all, whether a fibroid should be removed at all in a pregravid uterus in women of reproductive age - in whom subsequent pregnancies could occur-should be given due consideration by gynaecologists in terms of the indications for surgery, as well as counselling the women of the implications for labour and delivery.

We would urge readers to scrutinise Table 6, which is a highly informative tableau rich in clinical details, from which several clinical lessons can be drawn. The majority of the women had only 1 previous caesarean section ruptured intrapartum and presented with abnormal fetal heart traces. What is striking is that some had labours lasting as long as 22 hours, and almost 1 in 5 had features of cephalopelvic disproportion. Indeed cases 21 and 43 were both term VBAC cases, one of whom was induced with prostaglandin and both augmented with oxytocin. These are timely reminders that the cautionary messages by Lydon-Rochelle et al. about increased

\footnotetext{
${ }^{1}$ Department of Obstetrics and Gynaecology, Singapore General Hospital, Singapore

${ }^{2}$ Minimally Invasive Unit, KK Women's \& Children's Hospital, Singapore

Correspondence: Assoc Prof Lay Kok Tan, Department of Obstetrics and Gynaecology, Singapore General Hospital, 16 College Road, Block 6 Level 7 , Singapore 169854.

Email: tan.lay.kok@singhealth.com.sg
} 
uterine rupture rates with the use of prostaglandins and oxytocin should be heeded, and also that the intrapartum management of VBACs should not be managed along similar lines like the unscarred primiparous woman. The threshold for recourse to caesarean section when faced with poor progress of labour must be lower.

With a rising caesarean section being an almost universal phenomenon, the issue of counselling and managing women with previous caesarean sections is entrenched in daily clinical practice. While there are already many excellent guidelines on this, the authors should be congratulated for both providing a contemporaneous review on obstetric uterine ruptures in a Singapore context and also raising awareness about the risks posed by previous laparoscopic myomectomies. Another important lesson is the significant maternal risks when uterine rupture occurs, which tend to be less highlighted compared to the fetal risk. In the current medico-legal climate, the need for clear and transparent evidence-based counselling cannot be overemphasised.

\section{REFERENCES}

1. Tan SQ, Chen LH, Muhd Abdul Qadir B, et al. Risk factors and outcomes of uterine rupture in Singapore: Emerging trends. Ann Acad Med Singap 2021;50:5-15.

2. Lydon-Rochelle M, Holt VL, Easterling TR, et al. Risk of uterine rupture during labor among women with a prior cesarean delivery. N Engl J Med 2001;345:3-8.

3. Chew SY. Uterine rupture in labour. A 10-year review. Singapore Med J 1984;25:24-9.

4. Chen LH, Tan KH, Yeo GS. A ten-year review of uterine rupture in modern obstetric practice. Ann Acad Med Singap 1995;24:830-5.

5. Ryan GA, Nicholson SM, Morrison JJ. Vaginal birth after caesarean section: Current status and where to from here? Eur J Obstet Gynecol Reprod Biol 2018;224:52-7. 